\title{
Latest electroweak and Higgs physics results from LHC
}

\section{Sandra Kortner*it}

Max-Planck-Institut für Physik, Munich

E-mail: sandra@mppmu.mpg.de

\begin{abstract}
After the discovery of a Higgs boson, it is more important than ever to explore the electroweak sector of the Standard Model together with its mechanism responsible for the generation of weak boson masses. Major recent results from the measurements of electroweak and Higgs boson processes with the ATLAS and CMS detectors at the LHC are presented. Results are based on the full set of $p p$ collision data at centre-of-mass energies of 7 and $8 \mathrm{TeV}$, as well as on $13 \mathrm{TeV}$ data collected in 2015 .
\end{abstract}

XXIV International Workshop on Deep-Inelastic Scattering and Related Subjects

11-15 April, 2016

DESY Hamburg, Germany

\footnotetext{
*Speaker.

$\dagger$ on behalf of the ATLAS and CMS Collaboration
} 


\section{Introduction}

The exploration of the electroweak sector of the Standard Model (SM) with its electroweak symmetry breaking Higgs mechanism is an integral part of the experimental research in particle physics. Some of the key probes for new physics beyond the SM in this sector are the gauge boson self-couplings [1] accessible via multiboson production processes at the Large Hadron Collider (LHC). Furthermore, with the discovery of the new particle whose properties are compatible to those of a scalar Higgs boson [2, 3], the exact nature of the Higgs mechanism can now be examined in a unique way. The low energy effects of the new physics beyond the $\mathrm{TeV}$ scale can be parametrised as effective anomalous triple and quartic gauge couplings (aTGC and aQGC). The presence of such couplings would lead to an enhanced multiboson production. The aTGC parameters are probed in inclusive diboson production. The measurements of the diboson production via vector boson scattering and the triboson production provide constraints on the aQGC parameters. In order to test the consistency of the discovered Higgs boson with the state-of-the-art predictions of the SM Higgs sector, several crucial properties have to be precisely measured: the mass of the new particle, its spin and parity, as well as the strength and the structure of its couplings to SM particles. Complementary to these indirect searches for new physics, also direct searches for additional Higgs bosons predicted by theories beyond the SM are performed.

In the following, recent results from the studies of electroweak and Higgs boson interactions with ATLAS [4] and CMS [5] experiments at the LHC will be presented. Results are obtained from the full set of Run 1 proton-proton ( $p p$ ) collision data collected in 2011 and 2012 at centre-of-mass energies of 7 and $8 \mathrm{TeV}$, respectively, as well as from the Run 2 data collected at a centre-of-mass energy of $13 \mathrm{TeV}$ by the end of 2015 .

\section{Diboson production and limits on aTGC parameters}

The diboson production channels $W \gamma, Z \gamma, W W, W Z$ and $Z Z$ have been observed by both ATLAS and CMS experiments already in $p p$ collision data at $\sqrt{s}=7 \mathrm{TeV}$. More extensive studies of these processes have been performed recently with $8 \mathrm{TeV}$ data. In addition, the $W Z$ and $Z Z$ processes are explored for the first time in a new energy regime at a centre-of-mass energy of $13 \mathrm{TeV}$. The measured integrated total or fiducial production cross sections at 8 and $13 \mathrm{TeV}$ are summarised in Table 1. All measurements agree with SM expectations. The measurements with $13 \mathrm{TeV}$ data $[13,16,17]$ are currently limited by the statistical uncertainty. The highest measurement precision for each channel is reached with $8 \mathrm{TeV}$ data.

The $Z \gamma$ production cross section is measured in an extended fiducial region separately for the $Z$ bosons decaying into electrons or muons $[6,7]$ and into neutrinos [6, 8]. The measured inclusive cross section is sensitive to a $10 \%-20 \%$ higher theoretical prediction from the next-tonext-to-leading order (NNLO) calculation as compared to the next-to-leading order (NLO) result. The dependence on higher order corrections is significantly reduced in case of the exclusive production with no jets in the final state. A similar impact of NNLO calculations is also seen for the total inclusive $W W$ cross section [9,10], yielding a prediction which is about $9 \%$ higher than at the NLO. This improves the level of agreement with the data to better than $1.4 \sigma$. The highest precision is achieved in the measurement of the $W Z$ production cross section at $8 \mathrm{TeV}$ [11] by the ATLAS ex- 


\begin{tabular}{|c|c|c|c|c|}
\hline Process & & $\sigma_{\text {meas }}$ and $\sigma_{\text {theory }}$ & $\begin{array}{c}\sqrt{s} \\
{[\mathrm{TeV}]}\end{array}$ & Ref. \\
\hline $\begin{array}{c}\sigma(p p \rightarrow Z \gamma+X) \\
\quad \times B R(Z \rightarrow \ell \ell)\end{array}$ & $\begin{array}{l}\text { ATLAS } \\
\text { Theory } \\
\text { CMS } \\
\text { Theory }\end{array}$ & $\begin{array}{ll}1507 \pm 10(\text { stat } .)_{-73}^{+78}(\text { syst. })_{-28}^{+29}(\text { lumi. }) \mathrm{fb} & \\
1483_{-37}^{+19}(\text { syst. }) \mathrm{fb} & \text { (NNLO, fid.) } \\
2063 \pm 19 \text { (stat. }) \pm 98(\text { syst. }) \pm 54(\text { lumi. }) \mathrm{fb} & \\
2241 \pm 22 \text { (syst. }) \mathrm{fb} & \text { (NNLO, fid.) }\end{array}$ & 8 & [6] \\
\hline $\begin{array}{c}\sigma(p p \rightarrow Z \gamma+X) \\
\times B R(Z \rightarrow v v)\end{array}$ & $\begin{array}{l}\text { ATLAS } \\
\text { Theory } \\
\text { CMS } \\
\text { Theory }\end{array}$ & $\begin{array}{l}68 \pm 4(\text { stat. })_{-3.2}^{+3.3}(\text { syst. }) \pm 1(\text { lumi. }) \mathrm{fb} \\
81.4_{-2.2}^{+2.4}(\text { syst. }) \mathrm{fb} \\
52.7 \pm 2.1 \text { (stat. }) \pm 6.4(\text { syst. }) \pm 1.4(\text { lumi. }) \mathrm{fb} \\
\left.50.0_{-2.2}^{+2.4} \text { (syst. }\right) \mathrm{fb}\end{array}$ & 8 & [6] \\
\hline$\sigma(p p \rightarrow W W+X)$ & $\begin{array}{l}\text { ATLAS } \\
\text { Theory } \\
\text { CMS } \\
\text { Theory }\end{array}$ & 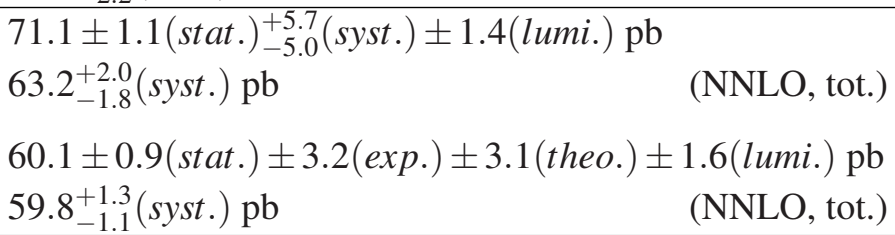 & 8 & {$[10]$} \\
\hline$\sigma(p p \rightarrow W Z+X)$ & $\begin{array}{l}\text { ATLAS } \\
\text { Theory } \\
\text { CMS } \\
\text { Theory } \\
\text { CMS } \\
\text { Theory }\end{array}$ & $\begin{array}{l}24.3 \pm 0.6 \text { (stat. }) \pm 0.6(\text { syst. }) \pm 0.4(\text { theo. }) \pm 0.5 \text { (lumi. }) \mathrm{pb} \\
21.0 \pm 1.6(\text { syst. }) \mathrm{pb} \\
24.61 \pm 0.76 \text { (stat. }) \pm 1.13 \text { (syst. }) \pm 1.08(\text { lumi. }) \mathrm{pb} \\
\left.21.91_{-0.88}^{+1.17} \text { (syst. }\right) \mathrm{pb} \\
\left.40.9 \pm 3.4 \text { (stat. })_{-3.3}^{+3.1}(\text { syst. }) \pm 0.4(\text { theo. }) \pm 1.3 \text { (lumi. }\right) \mathrm{pb} \\
\left.42.6_{-0.8}^{+1.6} \text { (syst. }\right) \mathrm{pb}\end{array}$ & 8 & $\begin{array}{c}{[12]} \\
{[13]}\end{array}$ \\
\hline$\sigma(p p \rightarrow Z Z+X)$ & $\begin{array}{l}\text { ATLAS } \\
\text { Theory } \\
\text { CMS } \\
\text { Theory } \\
\text { ATLAS } \\
\text { Theory } \\
\text { CMS } \\
\text { Theory }\end{array}$ & 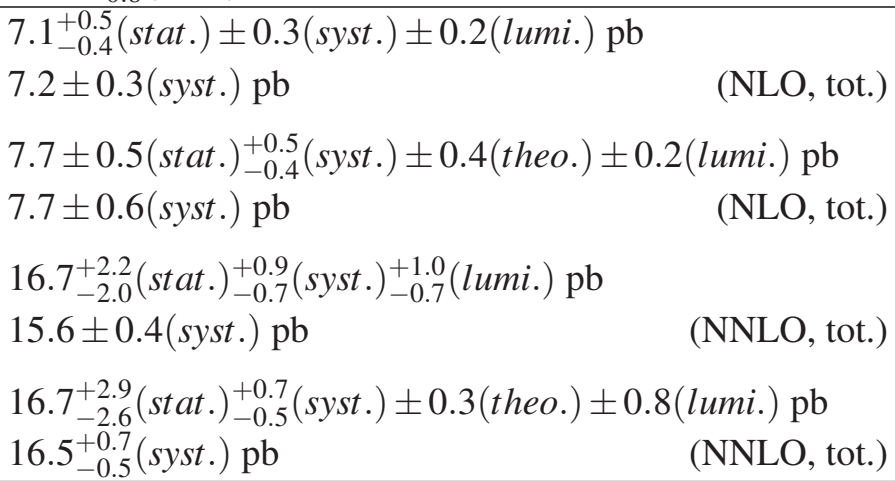 & 13 & [16] \\
\hline
\end{tabular}

Table 1: Measured and predicted integrated total (tot.) or fiducial (fid.) cross sections for the $Z \gamma, W W, W Z$, and $Z Z$ production at $\sqrt{s}=8$ and $13 \mathrm{TeV}$ at the LHC. Theory predictions are described in the corresponding ATLAS and CMS references.

periment. This is the first diboson cross section measurement in which the total relative experimental uncertainty of $4.2 \%$ is now lower than the present uncertainty of the NLO SM prediction. The slight difference between the measured and the predicted cross section may be compensated by the NNLO calculation [18], which is expected to increase the SM prediction in a similar way as in the $Z \gamma$ and $W W$ channels. The ratio of the $W^{+} Z$ to $W^{-} Z$ fiducial production cross sections is sensitive to the choice of the parton distribution function (PDF). Almost all systematic uncertainties cancel out in this measurement. The observed ratio of $1.51 \pm 0.08$ (stat.) \pm 0.01 (syst.) \pm 0.01 (lumi.) [11] 
agrees with the SM prediction and is dominated by the statistical uncertainty which is of the same order of magnitude as the estimated PDF uncertainty of the theory prediction.

In addition to integrated cross sections, the distributions of several kinematic variables are studied in each channel, probing the effects of the higher order perturbative QCD corrections or non-SM contributions to diboson production. A fair agreement between the shapes of measured unfolded differential cross section distributions and the NLO SM predictions from different Monte Carlo generators is observed for most of the variables. A global excess of events in the $W Z$ channel at $8 \mathrm{TeV}$ seems to be related to the region with neutrinos of low transverse momenta. The production of the photons with high transverse energy in the $Z \gamma$ channel is shown to be reproduced by the NNLO calculation while the NLO calculation significantly underestimates the data.

Selected detector-level kinematic distributions are employed in search for aTGCs. The transverse momentum of the leading lepton and the dilepton invariant mass in the $W W$ channel, as well as the transverse mass of the $W Z$ system in the $W Z$ channel are sensitive to the charged $W W \gamma$ and $W W Z$ aTGCs. The neutral aTGCs are probed by the distributions of the transverse photon energy and the four-lepton invariant mass in the $Z \gamma$ and $Z Z$ channels, respectively. The event yields in high energy tails of these distributions are expected to increase in the presence of aTGCs. The observed spectra are in agreement with the SM predictions and can be interpreted in terms of $95 \%$ confidence intervals for the corresponding aTGCs. The recent limits from $8 \mathrm{TeV}$ data are by a factor of two to three stronger than those from the $7 \mathrm{TeV}$ data [19].

\section{Vector boson scattering, triboson production and limits on aQGC parameters}

The diboson production via scattering of two vector bosons (VBS) is a key processes to probe the nature of the electroweak symmetry breaking. In the absence of the SM Higgs boson, the amplitude for the $W W$ scattering would increase with increasing centre-of-mass energy and ultimately violate unitarity. At the LHC, the VBS processes sensitive to aQGC parameters are realised through an interaction of vector bosons radiated from initial state quarks. This gives in a final state with two vector bosons and two jets $(V V j j)$. The jets from this purely electroweak process tend to be well separated in rapidity and have a large dijet invariant mass, allowing for discrimination from the QCD $V V j j$ production via strong interactions. The VBS processes cannot be distinguished from other electroweak $V V j j$ contributions, such that only a combined electroweak $V V j j$ signal is measured. The search for the electroweak $V V j j$ production has been performed with $8 \mathrm{TeV}$ data in various channels. First evidence was seen by the ATLAS experiment in the $W W j j$ channel with two leptons of same electric charge in the final state [20], with a similar study performed also by the CMS experiment [21]. Recently, also evidence for the electroweak $Z \gamma j j$ production has been presented by the CMS experiment [22] (see Fig. 1 (left)). The electroweak $W \gamma j j$ production is observed with a signal significance of $2.7 \sigma$ [23]. The measured fiducial cross sections are in good agreement with the SM predictions as shown in Table 2. The search for the electroweak $W Z j j$ production has been performed by the ATLAS experiment [11], yielding an upper limit on the fiducial production cross section of $0.63 \mathrm{fb}$ at $95 \%$ confidence level, while the SM prediction at NLO is $0.13 \pm 0.01 \mathrm{fb}$.

The anomalous quartic gauge couplings can be additionaly probed by the triboson production processes. The measuremed integrated fiducial cross sections are summarised in Table 2. The $Z \gamma \gamma$ 

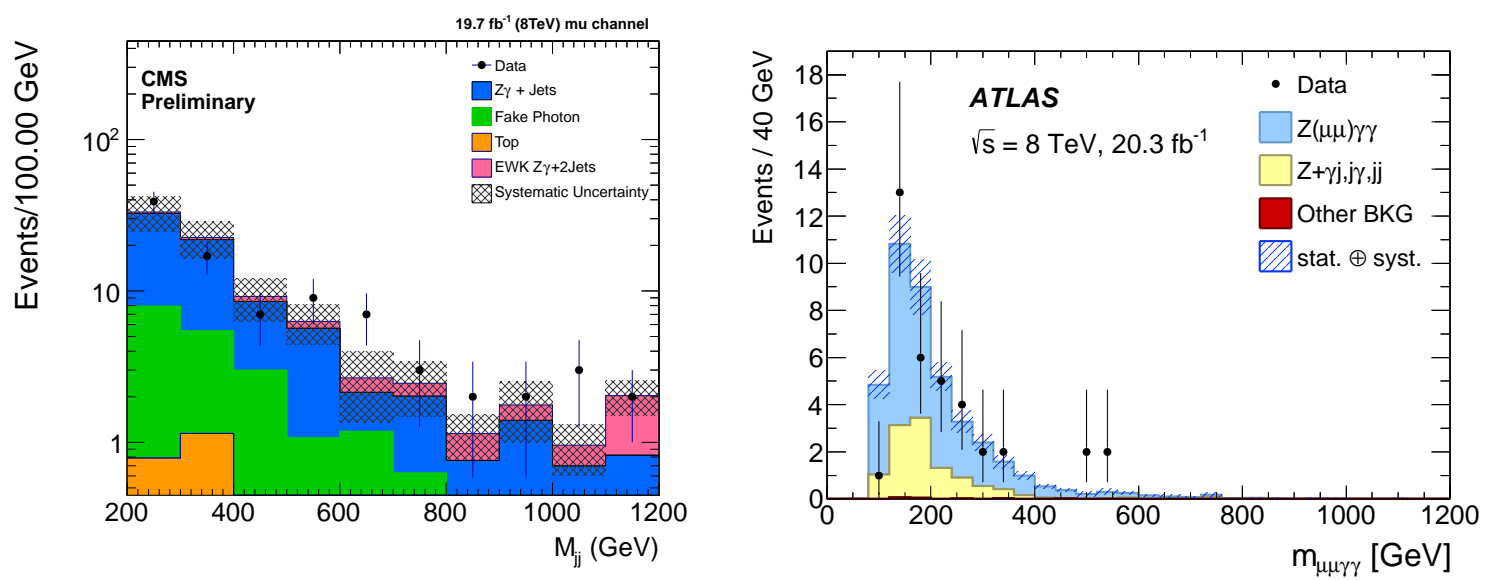

Figure 1: Distributions of the invariant dijet mass in the $Z \gamma j j$ channel (left) and the invariant $\mu \mu \gamma \gamma$ mass in the $Z \gamma \gamma$ channel (right). Only distributions for final states with muons are shown. Similar distributions are seen also in final states with electrons $[6,22]$.

\begin{tabular}{|c|l|lr|l|}
\hline Process & & $\sigma_{\text {meas }} \pm($ stat. $) \pm($ syst. $) \pm($ lumi. $)[\mathrm{fb}]$ & Ref. & $\sigma_{\text {theory }} \pm($ syst $)[\mathrm{fb}]$ \\
\hline$W W j j$ & ATLAS & $2.1 \pm 0.5 \pm 0.3$ & {$[20]$} & $1.52 \pm 0.11(\mathrm{NLO})$ \\
& CMS & $4.0_{-2.01}^{+2.4+1.1}$ & {$[21]$} & $5.8 \pm 1.2(\mathrm{NLO})$ \\
\hline$Z \gamma j j$ & CMS & $1.86_{-0.75}^{+0.89}{ }_{-0.27}^{+0.41} \pm 0.05$ & {$[22]$} & $1.26 \pm 0.12(\mathrm{LO})$ \\
\hline$W \gamma j j$ & CMS & $10.8^{+0.1} \pm 3.4 \pm 0.3$ & {$[23]$} & $6.1 \pm 1.2(\mathrm{LO})$ \\
\hline$Z \gamma \gamma$ & ATLAS & $5.07_{-0.68}^{+0.73}-_{-0.38} \pm 0.1$ & {$[6]$} & $3.70_{-0.11}^{+0.21}(\mathrm{NLO})$ \\
& CMS & $12.7_{1.1} \pm 1.4 \pm 1.8 \pm 0.3$ & {$[24]$} & $12.95 \pm 1.47$ (NLO) \\
\hline$W \gamma \gamma$ & ATLAS & $6.1_{-1.0}^{+1.1} \pm 1.2 \pm 0.2$ & {$[25]$} & $2.90 \pm 0.16(\mathrm{NLO})$ \\
& CMS & $6.0 \pm 1.8 \pm 2.3 \pm 0.2$ & {$[24]$} & $4.76 \pm 0.53$ (NLO) \\
\hline
\end{tabular}

Table 2: Measured and predicted integrated fiducial cross sections for the electroweak $W W j j, W \gamma j j, Z \gamma j j$ and the triboson $W \gamma \gamma$ and $Z \gamma \gamma$ production at $\sqrt{s}=8 \mathrm{TeV}$ at the LHC. Theory predictions are described in the corresponding ATLAS and CMS references.

process with leptonic $Z$ boson decays has been observed by both ATLAS and CMS experiments with a signal significance of $6.3 \sigma$ and $5.9 \sigma$, respectively [6,24]. A representative distribution of events in the signal region is shown in Fig. 1 (right). An evidence at the level of $3 \sigma$ has been seen by the ATLAS experiment also for the $W \gamma \gamma$ process [25], with a similar signal significance of $2.7 \sigma$ reported by the CMS experiment [24]. The search for the $W W \gamma$ and $W Z \gamma$ production has also been performed [26], setting an upper cross section limit which is at $95 \%$ confidence level about 3.4 times larger than the SM NLO prediction.

Quartic gauge couplings are probed by the most sensitive kinematic distribution in a given $V V j j$ or triboson channel: dilepton invariant mass $(W W j j)$, transverse momentum of the $W$ boson ( $W \gamma j j$ ), invariant mass of the $Z \gamma$ system $(Z \gamma j j)$, azimuthal angle between the $W$ and $Z$ boson and the sum of the lepton transverse momenta $(W Z j j)$, as well as the diphoton invariant mass and the transverse momentum of the leading photon $(W \gamma \gamma, Z \gamma \gamma, W W \gamma)$. No excess of events above the SM prediction is observed and the $95 \%$ confidence intervals are set for a number of aQGC 
parameters [19]. The sensitivity reached with triboson processes is about a factor of ten worse than from the electroweak $V V j j$ measurements.

\section{Properties of the discovered Higgs boson}

A strong effort has been made to investigate the properties of the new Higgs boson [2, 3] using the entire Run 1 data. The mass of the new particle, which determines the Higgs boson production and decay rates in the SM, has been measured with high precision. Signal strengths, i.e. signal event yields relative to their SM predictions, are measured in all accessible production and decay modes, allowing for the study of the Higgs boson couplings to SM particles. Finally, the structure of the couplings to gauge bosons is examined based on the kinematic properties of the production and decay products.

An essential ingredient for all tests of the SM Higgs sector is the precise mass value of the discovered Higgs boson. The mass measurement is performed independently in the diphoton and four-lepton Higgs decay channels, the only two final states with a high mass resolution of better than $1 \mathrm{GeV}$ and good signal sensitivity. The results of individual ATLAS and CMS measurements and of their combination are shown in Fig. 2 [27]. The combined mass value of

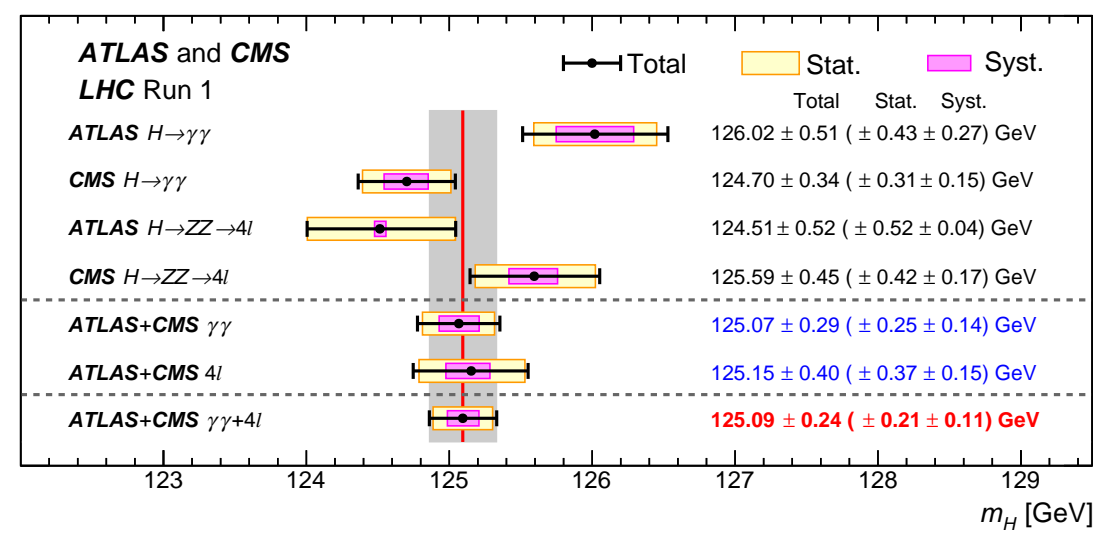

Figure 2: Summary of Higgs boson mass measurements from the individual analyses of 7 and $8 \mathrm{TeV}$ data by ATLAS and CMS and from the combined analysis [27].

$m_{H}=125.09 \pm 0.21$ (stat.) \pm 0.11 (syst.) $\mathrm{GeV}$ is obtained. The already very high measurement precision of $0.2 \%$ is dominated by a statistical uncertainty and will further improve with additional Run 2 data.

The compatibility of the discovered particle with the SM Higgs boson is foremost investigated by measuring the signal strengths $\mu$ relative to the corresponding SM predictions in the dominant decay modes: $\gamma \gamma, Z Z, W W, b b, \tau \tau, \mu \mu$. The rates are measured separately for the main Higgs boson production processes: the dominant production via gluon fusion $(\mathrm{ggF})$, vector boson fusion (VBF) with an about twelve times lower predicted cross section, and the even rarer production in association with a vector boson $(\mathrm{VH})$ or with a pair of top quarks $(t \bar{t} H)$. The ATLAS and CMS measurements in individual channels are combined to increase the sensitivity to a given Higgs 
production or decay mode [28]. Figure 3 shows the measured signal strength of individual production (decay) modes, assuming the SM values for the Higgs boson branching fractions (production cross sections). The $p$-value of the compatibility between the data and the SM predictions is $24 \%$ and $75 \%$ for the production and decay signal strengths, respectively. A combined signal strength
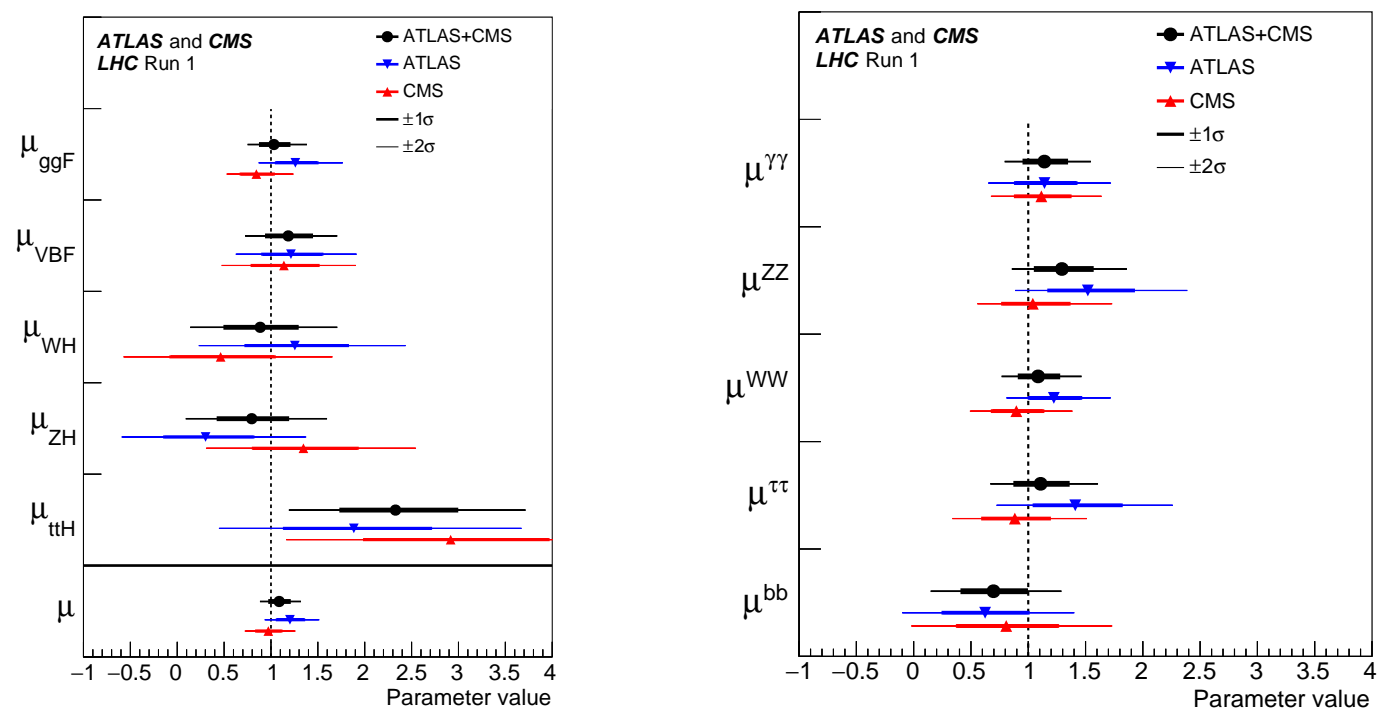

Figure 3: The measured production (left) and decay (right) signal strengths $\mu^{i}$ relative to the SM prediction for the combination of ATLAS and CMS data. Also shown are the results from each experiment [28].

of $1.09 \pm 0.07$ (stat.) \pm 0.04 (exp.) \pm 0.03 (thbg.) ${ }_{-0.06}^{+0.07}$ (thsig.) is obtained [28], in good agreement with the SM prediction. The systematic uncertainty is dominated by the precision in the theoretical prediction of the SM Higgs boson production cross section (thsig.), while the uncertainty on the background prediction (thbg.) is two times smaller. The Higgs boson production via gluon fusion and the Higgs boson decays into gauge bosons have been observed with high significance independently by the two experiments. The combination of ATLAS and CMS data leads in addition to the clear observation of VBF production and $H \rightarrow \tau \tau$ decays with signal significance of $5.4 \sigma$ and $5.5 \sigma$, respectively. The significance for the $V H$ prodcution is above $3 \sigma$. The combined significance for the $t \bar{t} H$ process is $4.4 \sigma$, whereas only $2.0 \sigma$ is expected, corresponding to a measured excess of events of $2.3 \sigma$ with respect to the SM prediction. The signal strengths measured in each individual channel can be expressed in terms of the Higgs boson couplings relative to the SM prediction. The ratios of coupling stregths are measured in a model-independet way with a precision of $10 \%-20 \%$, showing a good agreement with the SM predictions. The potential presence of physics beyond the $\mathrm{SM}$ is also probed using specific assumptions on the presence of new particles in $\mathrm{ggF}$ production and $\gamma \gamma$ decay loops, or in direct Higgs boson decays. Under the assumption that there are no new particles appearing in the loops or in direct decays, the $p$-value of the compatibility between the data and the SM predictions is $74 \%$.

The kinematic and angular correlations of the Higgs boson production and decay products provide information on the spin and the parity of the new particle. Extensive tests of various fixed spin-parity $J^{C P}$ hypotheses $[29,30]$ exclude at more than $99.9 \%$ confidence level all alternative models in favour of the SM hypothesis of a spin $0^{++}$particle. Possible small admixtures of non- 
SM CP states are now probed by measuring the tensor structure of the Higgs couplings to weak bosons. The strongest limits on the non-SM CP-even and CP-odd (CP-violating) couplings at 95\% confidence level are set based on the topology of the Higgs decays into weak bosons, $H \rightarrow$ $V V[29,30]$. Additional sensitivity is gained from the VBF $H \rightarrow \tau \tau$ channel using the matrixelement observable constructed from the kinematic properties of the Higgs boson and the two tagging jets from the VBF production [31]. There is currently no sensitivity to set limits at $95 \%$ confidence level, but the limits obtained at $68 \%$ confidence level are about a factor of ten stronger than the corresponding results from $H \rightarrow V V$ decays. The search for the CP-odd couplings is also performed in the $\mathrm{VH} H \rightarrow b \bar{b}$ channel which on its own has currently no strong sensitivity. However, the combination with the $H \rightarrow V V$ measurements strongly improves the current limit if one assumes that the ratio of the Higgs couplings to top- and bottom-quarks follows the SM prediction.

Although still limited by the statistical uncertainty, first measurements with $13 \mathrm{TeV}$ data have also been performed for a few key decay channels. The cleanest final states with Higgs boson decays into two photons and into four leptons have been studied by both ATLAS and CMS experiments $[33,34,35,36]$. Figure 4 shows the resulting invariant diphoton and four-lepton mass distributions observed by the ATLAS and CMS experiments, respectively. While no signal is observed
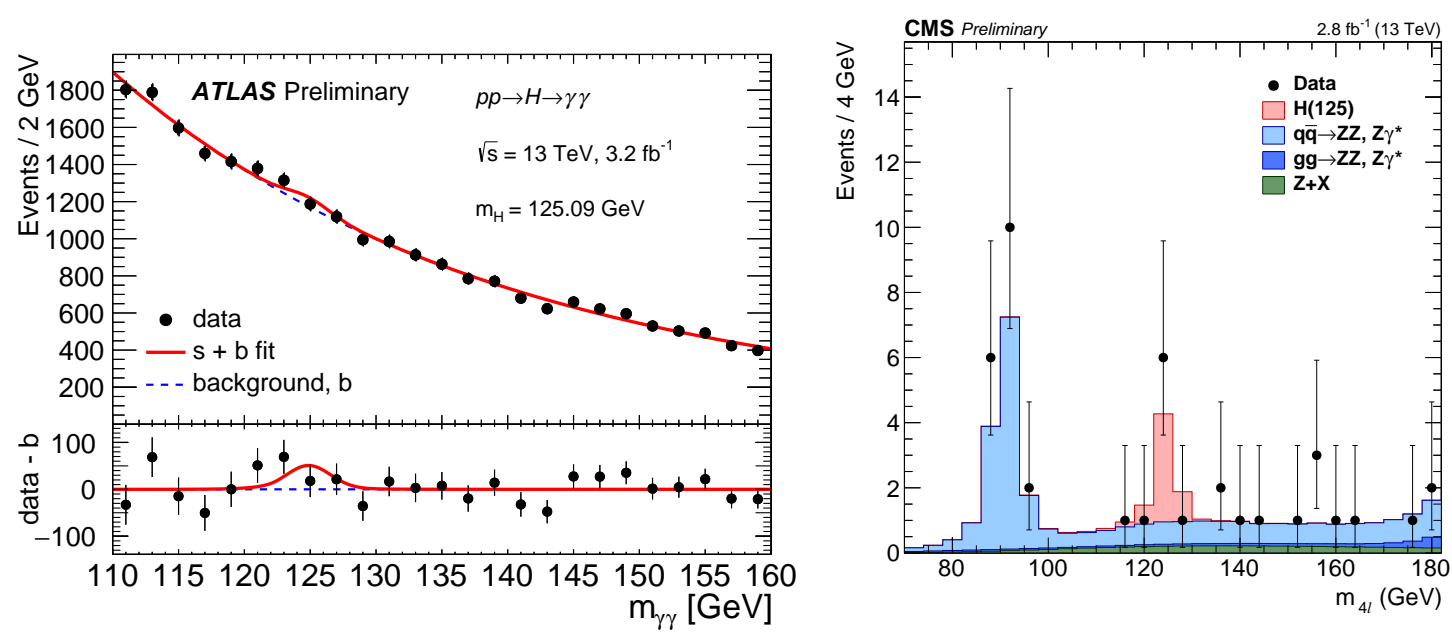

Figure 4: Diphoton $m_{\gamma \gamma}$ (left) and the four-lepton $m_{4 \ell}$ (right) invariant mass spectra observed in the $13 \mathrm{TeV}$ data in search for the SM Higgs boson [34, 35].

in the four-lepton channel by the ATLAS experiment due to statistical fluctuations in data, the CMS experiment observes a signal with a significance of $2.5 \sigma$. The signal significances observed in the diphoton channel by the ATLAS and CMS experiments are $1.7 \sigma$ and $2.5 \sigma$, respectively. The signal strengths and the cross sections measured in individual channels and for the combination of the two ATLAS measurements [37] are compatible with the SM predictions. Motivated by a slight excess of $t \bar{t} H$ events at the level of $2.3 \sigma$ observed with Run 1 data, a first look is also given to this production process in $13 \mathrm{TeV}$ data. The signal strengths of $3.8_{-3.6}^{+4.5},-2.0_{-1.8}^{+1.8}$ and $0.6_{-1.1}^{+1.4}$ are measured in final states with Higgs boson decays into two photons [36], two bottom-quarks [38] or into several leptons [39], respectively, with no significant deviations from the SM predictions. 


\section{Search for new scalar resonances}

The indirect search for new physics via precision measurements of Higgs boson properties is complemented by direct searches for new Higgs bosons predicted by a number of theories beyond the SM. Over the past two years, a devoted endeavor has been made to search for signatures of new charged or neutral Higgs bosons decaying into heavy leptons and quarks or gauge boson pairs in a broad range of theories beyond the SM. No significant sign of new physics has been seen in any of these searches. Only recently, an intriguing excess of diphoton events is observed in Run 2 data at a diphoton mass of about $750 \mathrm{GeV}$ by both ATLAS and CMS experiments in their search for scalar and spin-2 resonances (see Fig. 5) [40, 41]. The excess of events seen by the ATLAS experiment is
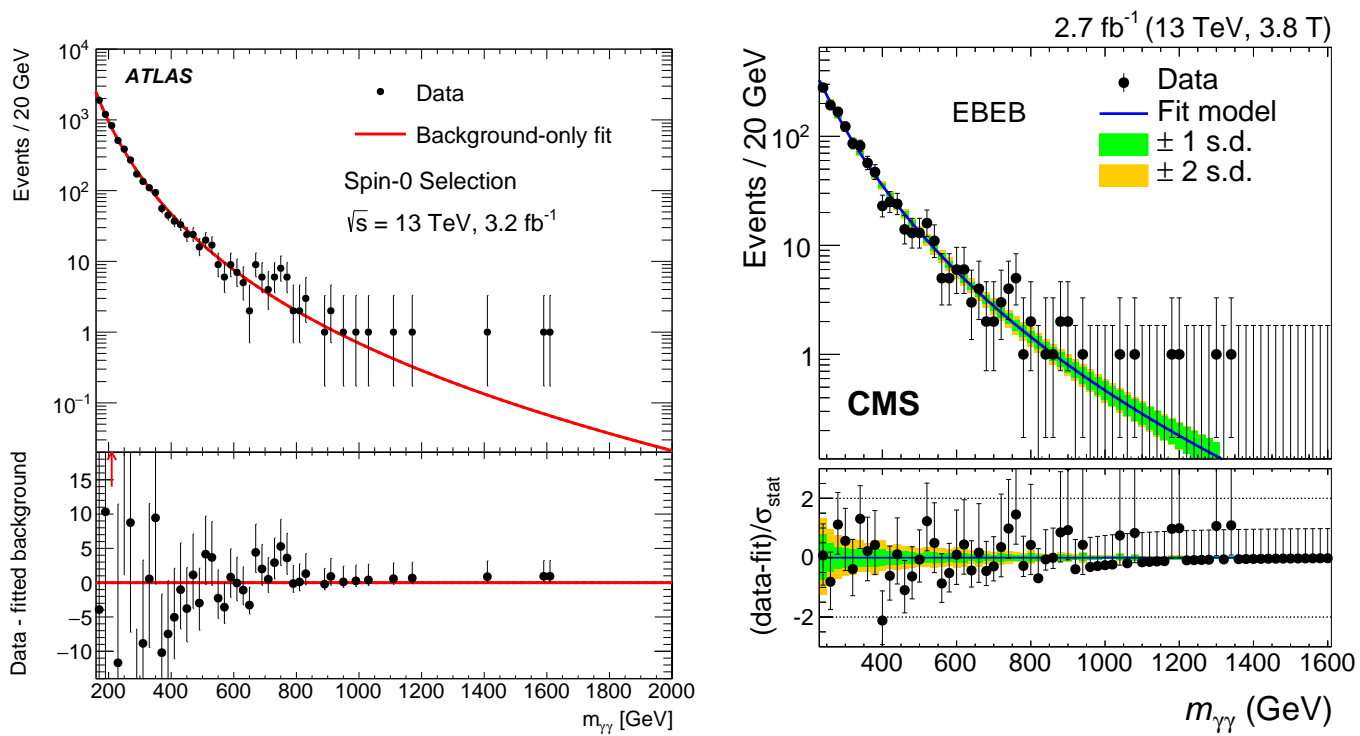

Figure 5: Invariant mass of the diphoton candidates observed by the ATLAS (left) and CMS experiment (right) with the best background-only fit. The difference between the data and this fit is shown in the bottom panel. EBEB stands for events with both photons in the barrel electromagnetic calorimeter of CMS [40, 41].

most compatible with a relatively broad resonance of $45 \mathrm{GeV}$ width, while the CMS data prefer the hypothesis of a narrow resonance. The local significance of the ATLAS excess is $3.9 \sigma(3.8 \sigma)$ for a spin- 0 (spin-2) resonance, with a global significance of $2.1 \sigma$ for both spin hypotheses. The local significance observed by the CMS experiment is $3.4 \sigma$ after the combination of 8 and $13 \mathrm{TeV}$ data. The corresponding global significance is $1.6 \sigma$. More data are required to determine the origin of this result.

\section{Summary}

In summary, the full set of Run 1 data allows for extensive tests of the electroweak sector of the SM. The electroweak data are consistent with the SM and sensitive to NNLO QCD corrections. An evidence for the VBS and triboson production has been seen in several channels and competitive constraints are set on aTGC and aQGC parameters. The properties of the discovered Higgs boson are thoroughly tested and shown to be consistent with the SM predictions. A wide range of 
direct searches for new Higgs particles is also performed. The future Run 2 data are expected to significantly extend the physics reach of these measurements.

\section{References}

[1] M. Baak et al., Community Summer Study 2013: Snowmass on the Mississippi (CSS2013) Minneapolis, MN, USA, July 29-August 6, 2013, arXiv:1310.6708 [hep-ph].

[2] ATLAS Collaboration, Phys. Lett. B716 (2012) 1.

[3] CMS Collaboration, Phys. Lett. B716 (2012) 30.

[4] ATLAS Collaboration, JINST 3 S08003.

[5] CMS Collaboration, JINST 3 (2008) S08004.

[6] ATLAS Collaboration, Phys. Rev. D 93, 112002 (2016).

[7] CMS Collaboration, JHEP 04 (2015) 164.

[8] CMS Collaboration, arXiv:1602.07152 [hep-ex], CERN-EP-2016-007 (February 2016), submitted to Phys. Lett. B.

[9] ATLAS Collaboration, arXiv:1603.01702 [hep-ex], CERN-PH-EP-2015-323 (March 2016), submitted to JHEP.

[10] CMS Collaboration, arXiv:1507.03268 [hep-ex], CERN-PH-EP-2015-122 (July 2015), submitted to Eur. Phys. J. C.

[11] ATLAS Collaboration, Phys. Rev. D 93, 092004 (2016).

[12] CMS Collaboration, CMS PAS SMP-12-006 (July 2013), URL: https://cds.cern.ch/record/1564318/files/SMP-12-006-pas.pdf.

[13] CMS Collaboration, CMS PAS SMP-16-002 (April 2016), URL: https://cds.cern.ch/record/2144880/files/SMP-16-002-pas.pdf.

[14] ATLAS Collaboration, ATLAS-CONF-2013-020 (March 2013), URL: https://cds.cern.ch/record/1525555/files/ATLAS-CONF-2013-020.pdf.

[15] CMS Collaboration, Phys. Lett. B740 (2015) 250.

[16] ATLAS Collaboration, arXiv:1512.05314 [hep-ex], CERN-PH-EP-2015-318 (March 2016), submitted to Phys. Rev. Lett.

[17] CMS Collaboration, CMS PAS SMP-15-005 (March 2016), URL: https://cds.cern.ch/record/2114822/files/SMP-15-005-pas.pdf.

[18] M. Grazzini, S. Kallweit, D. Rathlev, M. Wiesemann, arXiv:1604.08576 [hep-ph] (April 2016).

[19] URL: https://twiki.cern.ch/twiki/bin/view/CMSPublic/PhysicsResultsSMPaTGC.

[20] ATLAS Collaboration, Phys. Rev. Lett. 113, 141803 (2014).

[21] CMS Collaboration, Phys. Rev. Lett. 114, 051801 (2015).

[22] CMS Collaboration, CMS PAS SMP-14-018 (September 2015), URL: https://cds.cern.ch/record/2048148/files/SMP-14-018-pas.pdf.

[23] CMS Collaboration, CMS PAS SMP-14-011 (January 2016), URL: https://cds.cern.ch/record/2124432/files/SMP-14-011-pas.pdf. 
[24] CMS Collaboration, CMS PAS SMP-15-008 (February 2016),

URL: https://cds.cern.ch/record/2130360/files/SMP-15-008-pas.pdf.

[25] ATLAS Collaboration, Phys. Rev. Lett. 115, 031802 (2015).

[26] CMS Collaboration, Phys. Rev. D 90, 032008 (2014).

[27] ATLAS and CMS Collaborations, Phys. Rev. Lett. 114 (2015) 191803.

[28] ATLAS and CMS Collaborations, arXiv:1606.02266 [hep-ex], CERN-EP-2016-100 (June 2016), submitted to JHEP.

[29] ATLAS Collaboration, Eur. Phys. J. C 75 (2015) 476.

[30] CMS Collaboration, Phys. Rev. D 92 (2015) 012004.

[31] ATLAS Collaboration, arXiv:1602.04516 [hep-ex], CERN-EP-2016-002 (February 2016).

[32] CMS Collaboration, arXiv:1602.04305 [hep-ex], CERN-PH-EP-2015-331 (February 2016), submitted to Phys. Lett. B.

[33] ATLAS Collaboration, ATLAS-CONF-2015-059 (December 2015), URL: http://cds.cern.ch/record/2114825/files/ATLAS-CONF-2015-059.pdf.

[34] CMS Collaboration, CMS PAS HIG-15-004 (March 2016), URL: https://cds.cern.ch/record/2139978/files/HIG-15-004-pas.pdf.

[35] ATLAS Collaboration, ATLAS-CONF-2015-060 (December 2015), URL: http://cds.cern.ch/record/2114826/files/ATLAS-CONF-2015-060.pdf.

[36] CMS Collaboration, CMS PAS HIG-15-005 (March 2016), URL: https://cds.cern.ch/record/2140979/files/HIG-15-005-pas.pdf.

[37] ATLAS Collaboration, ATLAS-CONF-2015-069 (December 2015), URL: http://cds.cern.ch/record/2114841/files/ATLAS-CONF-2015-069.pdf.

[38] CMS Collaboration, CMS PAS HIG-16-004 (March 2016), URL: https://cds.cern.ch/record/2139578/files/HIG-16-004-pas.pdf.

[39] CMS Collaboration, CMS PAS HIG-15-008 (March 2016), URL: https://cds.cern.ch/record/2141078/files/HIG-15-008-pas.pdf.

[40] ATLAS Collaboration, arXiv:1606.03833 [hep-ex], CERN-EP-2016-120 (June 2016), submitted to JHEP.

[41] CMS Collaboration, arXiv:1606.04093 [hep-ex], CERN-EP-2016-154 (June 2016), submitted to Phys. Rev. Lett. 